\title{
De la narratologie à la narrativité, et retour
}

Bilan et perspectives de la théorie greimassienne

From Narratology to Narrativity, and Back. Assessment and perspectives of

Greimassian theory

\section{Denis Bertrand}

\section{CpenEdition \\ Journals}

\section{Édition électronique}

URL : http://journals.openedition.org/pratiques/6122

DOI : $10.4000 /$ pratiques. 6122

ISSN : 2425-2042

\section{Éditeur}

Centre de recherche sur les médiations (CREM)

\section{Référence électronique}

Denis Bertrand, « De la narratologie à la narrativité, et retour », Pratiques [En ligne], 181-182 | 2019, mis en ligne le 30 juin 2019, consulté le 20 juillet 2019. URL : http://journals.openedition.org/ pratiques/6122; DOI : 10.4000/pratiques.6122

Ce document a été généré automatiquement le 20 juillet 2019.

(c) Tous droits réservés 


\title{
De la narratologie à la narrativité, et retour
}

\author{
Bilan et perspectives de la théorie greimassienne \\ From Narratology to Narrativity, and Back. Assessment and perspectives of \\ Greimassian theory
}

Denis Bertrand

\section{Bilan}

\section{La sémiotique narrative : l'oubliée de la nouvelle narratologie}

1 Plus que tout autre, le concept de "narrativité » est emblématique de la sémiotique greimassienne ${ }^{1}$. Les instruments analytiques qui en découlent paraissent désormais faire partie des acquis incontestés de cette théorie, du moins pour les chercheurs qui s'en réclament. Or, une raison circonstancielle et une raison théorique nous invitent aujourd'hui à reconsidérer le statut de ce concept. La première nourrit la seconde : elle nous amène en effet à réexaminer les fondements de la narrativité dans le cadre global des principes qui assurent à la sémiotique son statut de science du langage, et qui expliquent qu'elle se soit peu à peu détachée de la narratologie.

2 La circonstance qui est à l'origine de cette réflexion est la résurgence inattendue de cette narratologie dans les débats actuels des sciences humaines en France. On sait que les études sur la nécessité d'adosser à un bon récit tout discours promotionnel - en politique comme en marketing - sont depuis longtemps présentes dans les pays anglo-saxons. Mais c'est le succès du mot d'ordre de «storytelling " qui a permis à cette narratologie de refaire surface en France. Le mot y a été acclimaté par la publication en 2007 du livre de C. Salmon, Storytelling. La machine à fabriquer des histoires et à formater les esprits. Plutôt que de théorie à proprement parler, il s'agit d'une réflexion de moraliste sur l'illusionnisme narratif et son pouvoir persuasif de manipulation. L'auteur observe que le développement du paradigme narratif « remplace le raisonnement rationnel» dans tous les domaines 
sociaux, économiques et politiques. Il prend acte du triomphe de ce paradigme au sein d'un débat qui remonte loin, puisqu'il agitait déjà le milieu des sciences du langage au cours des années 1970: il opposait, comme deux modèles concurrents susceptibles de fonder l'intelligibilité et l'efficacité du discours, d'un côté le modèle argumentatif issu de la rhétorique et relancé par les travaux qui vont de C. Perelman à ceux du Centre de recherches sémiologiques de Neuchâtel (autour de J.-B. Grize), et de l'autre le modèle narratif issu pour l'essentiel de la sémiotique greimassienne. La revue Pratiques s'est souvent fait l'écho indirect de cette disputatio en accordant ses colonnes tantôt aux narrativistes (greimassiens ou non), tantôt aux argumentativistes (disciples ou non de logiciens comme J.-B. Grize ou de pragmaticiens comme O. Ducrot). Quoi qu'il en soit, selon C. Salmon, les jeux sont faits et, du moins dans le champ de la communication sociale, la narration l'a emporté haut la main. Son succès constituerait même, comme on peut le lire sur la note de présentation de l'ouvrage, «un incroyable hold-up sur l'imagination des humains " à qui il imposerait ni plus ni moins un "nouvel ordre narratif ». De fait, le mot «storytelling », désormais francisé, est devenu un des mots-clés du métalangage ordinaire en cette deuxième décennie du xxI ${ }^{\mathrm{e}}$ siècle. Il est dans toutes les bouches, depuis les chroniqueurs médiatiques jusqu'aux conseillers en communication.

Or le retour de la narratologie se manifeste également, de manière plus académique, par l'organisation de rencontres internationales sur le récit, réunissant des centaines d'intervenants : exemple parmi d'autres, le congrès " Narrative matters. Récit et savoir ", réalisé par l'Université américaine de Paris avec l'Université Paris Diderot, en juin 2014. Mais il y a aussi ceux qu'organisent régulièrement la European Narratology Association, ou l' International Society for the Study of Narrative. On ne sait si « Narrative matters » doit être compris comme "Matériaux narratifs » ou comme «Le récit, ça compte », les deux sans doute. Quoi qu'il en soit, le congrès « Récit et savoir » était résolument transdisciplinaire : il réunissait des chercheurs de toutes les sciences humaines et sociales - psychologie, psychanalyse, sociologie, anthropologie, histoire, philosophie, sciences du langage, études littéraires, études féministes et études de genre, éducation, médecine, santé et action sociale, biologie, droit, science des religions, informatique, visual studies... -, afin de réfléchir sur « la question des puissances épistémiques, parfois controversées, du récit ». Il s'agissait, en somme, de prendre acte de la prééminence du récit sur l'argumentation au regard de la cognition. De sorte que, accepté comme une donnée immédiate, naturelle et spontanée du discours, le récit étendrait son ombre matricielle - ou sa lumière - sur tous les domaines de la connaissance.

4 La principale référence théorique convoquée est celle de D. Polkinghorne avec son ouvrage Narrative Knowing and the Human Sciences (1988). L'argument central de ce livre indique clairement l'orientation de son projet de narratologie cognitive : le récit, écrit l'auteur,

constitue un schème au moyen duquel les êtres humains donnent une signification à leur expérience de la temporalité et à leurs actions personnelles. [...] Les significations narratives fournissent un cadre permettant de comprendre les événements passés de la vie d'une personne et de projeter les actions futures ${ }^{3}$. (ibid., p. 11)

Cet argument reprend en réalité la thèse centrale soutenue par P. Ricœur dans son monumental travail Temps et récit (Ricœur, 1983, 1984, 1985). Le philosophe, comme on le sait, confère au récit la fonction phénoménologique de donner une forme, une lisibilité et une orientation finalisée au divers évènementiel de l'expérience vécue qui serait chaotique sans lui ; de plus, il offre à la temporalité et à la mémoire une structure 
d'accueil qui les rend saisissables. La fonction essentielle du récit est donc de fournir une " synthèse de l'hétérogène » en imposant, à travers ses configurations ordonnées (du seul fait de leur "mise en signes" - verbaux ou non), un pouvoir constitutif de « refiguration » de notre expérience vive, immergée dans les apories du temps et rendue alors intelligible, par la force d'ordonnancement du discours narratif.

Or, et c'est là qu'intervient la raison théorique dont nous parlions plus haut, ce retour de la narratologie ne fait état d'aucun voisinage, d'aucune parenté, d'aucune filiation, en dehors de celle, indirecte, à P. Ricœur, avec les recherches développées en Europe, et particulièrement en France, dans le domaine de la théorie narrative depuis les années 1960 et 1970 : rien sur les travaux de R. Barthes, de T. Todorov, de G. Genette et à fortiori sur ceux, plus confidentiels peut-être, d'A. J. Greimas. Il ne s'agit évidemment pas ici de prendre position ou d'émettre un jugement sur cette absence, ou cette occultation. Pas de condamnation, ni de plainte sur le caractère infranchissable de frontières intellectuelles qui ont, dans l'ordre symbolique et épistémologique, l'étendue d'un océan. Il s'agit plutôt d'en comprendre les raisons. On peut sans doute les trouver dans une différence d'approche où le pragmatisme des effets (le récit, ça fait quoi ?) l'emporte sur l'immanentisme des formes (le récit, c'est fait comment?), où le comprendre mieux a la priorité sur l'expliquer plus, où la philosophie du langage en acte domine le structuralisme linguistique et discursif. Mais, s'agissant ici des "théories du récit en débat», on s'intéressera davantage aux raisons internes qui ont pu conduire les sémioticiens, à la suite d'A.J. Greimas, à abandonner la narratologie au profit de la narrativité, et la narrativité au profit d'une sémiotique du sensible, de l'énonciation et des interactions.

\section{Du sens et du narratif : la transformation}

7 L'examen de la composition de Sémantique structurale (Greimas, 1966) et la lecture de l'introduction à Du sens (Greimas, 1970) elle aussi intitulée, en une sorte de redondance obstinée, «Du sens », sont d'emblée éclairants. Dans le premier, tout commence par le minutieux travail de mise en place d'une analyse sémique scientifiquement fiable. Celle-ci est ensuite étendue, avec l'isotopie, à la dimension du discours, et conduit à la découverte de la «pluri-isotopie » comme condition, sinon d'existence du moins de manifestation, de ce fameux sens qu'on cherche à saisir et à décrire. Et enfin, ce n'est que dans un troisième temps que la structuration narrative de cet objet fait son apparition, à travers la relecture de la Morphologie du conte de V. Propp et la mise en place, par une réduction drastique des fonctions proppiennes, du fameux modèle actantiel (Destinateur-Sujet-Destinataire; Adjuvant-Opposant). Le narratif n'est donc pas un objet donné dès le départ. Il émerge peu à peu, et finit par s'imposer dans la logique d'une interrogation sur les conditions de saisie du sens.

C'est cette même question qui ouvre le deuxième texte, l'introduction à Du sens. Texte admirable, véritable récit cognitif, où l'état initial de manque où se trouve le chercheur "Il est extrêmement difficile de parler du sens et d'en dire quelque chose de sensé » (Greimas, 1970, p. 7) - le conduit, pour tenter de le liquider, à explorer diverses pistes, à risquer de multiples hypothèses, à se confronter à des épreuves que sanctionneront d'inévitables échecs, jusqu'à la découverte finale, incertaine et provisoire, mais assez ferme cependant, que le sens est contenu dans ce mot : «transformation ». De là viendra la narrativité, le cœur syntaxique de tout récit étant le passage d'un état à un autre état, de la pauvreté à la richesse, de l'humiliation à la gloire, bref, une transformation. 
C'est ainsi qu'A. J. Greimas fut le seul, parmi ses compagnons-chercheurs de renom, à intégrer l'«analyse structurale du récit» à un dispositif théorique de plus grande ampleur et à reconnaitre le narratif comme une condition générale de saisie de la signification. La narrativité joue en effet un rôle proprement crucial dans la théorie générale du langage, bien loin des seules vertus du récit dans la variété de ses formes. Lorsqu'on réexamine aujourd'hui l'ouvrage de référence, Sémiotique. Dictionnaire raisonné de la théorie du langage (Greimas \& Courtés, 1979), on est frappé par la présence transversale, et presque invasive, de la narrativité dans un grand nombre de ses entrées $\mathrm{y}$ compris dans celles qui n'ont à première vue rien à voir avec le récit. C'est le cas, par exemple, de l'entrée "Immanence ", où la catégorie immanence/transcendance est utilisée, de manière sans doute limitative, pour rendre compte des statuts différenciés des actants Sujet (héros) et Destinateur (instance d'autorité, qui mandate le héros et sanctionne ses actions) (Greimas \& Courtés, 1979, p. 182, « Immanence »). Mais c'est aussi le cas de bien d'autres notions comme celles de sujet, de modalité, d'aspectualité, de mode d'existence, d'énonciation elle-même, toutes redevables, dans leur définition, de l'incidence narrative.

Voici donc que le narratif émerge d'une interrogation générale sur le sens et sur ses conditions de manifestation. Et voici qu'en réponse à cette question il devient la cheville ouvrière de ce sens éprouvé, perçu, compris et partagé. Car si le sens est transformation, la transformation maitresse est bien celle du récit. C'est elle qui charpente les discours. Et elle parle à nos imaginaires qu'elle structure en retour. Grammaire transformationnelle, la syntaxe narrative, née de cet impératif de transformation, se situe donc bien en amont du récit proprement dit. D'un côté, elle rencontre les intuitions narratives et ses mises en scènes figuratives à travers toutes les histoires que nous nous racontons; et de l'autre, elle retourne à l'abstraction qui est son foyer pour devenir une structuration modale et actantielle sous-jacente à tout discours. C'est ainsi qu'on peut découvrir le point de jonction de la sémiotique narrative avec la phénoménologie du temps de P. Ricœur : le divers confus de l'expérience vécue est ordonné par la mise en intrigue, dit le philosophe ; il est mis en ordre par les structures narratives, dit le sémioticien.

\section{Une approche du discours ordonnée par le paradigme narratif : l'ossature modale de la narrativité}

11 Envisagée dans son développement, la construction de la grammaire narrative peut être comprise comme un approfondissement progressif de l'analyse à partir des fonctions proppiennes, c'est-à-dire comme un long et progressif travail d'abstraction, de réduction et de détachement des données figuratives du conte merveilleux qui en a été le corpus fondateur. Le premier acte a été de réduire les trente et une fonctions de V. Propp, à la fois combinatoire et trame de tout récit possible, à un schéma narratif de quatre séquences (contrat > compétence > performance > reconnaissance), puis de ramener celles-ci aux trois grandes sphères sémiotiques de la manipulation (englobant le contrat et la formation de la compétence du sujet), de l'action (cœur de la transformation) et de la sanction (positive ou négative). Une seconde réduction porte sur les dramatis personae qui, identifiés comme actants, conjuguent le double statut de "personnages » et d'objetslangage en tant que figures syntaxiques (sujet/objet et destinateur/destinataire). Ces actants, affectés de valeurs positives et/ou négatives, voient leurs relations modélisées dans des syntagmes élémentaires, les « programmes narratifs » transformationnels (de la conjonction à la disjonction et inversement). L'ossature d'une grammaire narrative de 
portée générale est ainsi mise en place. La narrativité est née, délaissant momentanément la narratologie et faisant d'elle une branche spécifique de son champ d'étude - celle des variations culturelles des récits, à travers la diversité de leurs genres, de leurs formes, de leurs stratégies.

C'est alors qu'est opérée une nouvelle réduction sur les actants, ces éléments moteurs de la dynamique narrative, par la modalité. Celle-ci, doublement inspirée des logiques modales et des modalisations linguistiques dont elle forme une synthèse, vient s'installer au cœur même de l'actant. Il ne reçoit plus une définition extérieure à lui-même, d'ordre thématique - telle que le dieu souverain, le héros, le traitre... - mais interne et définissant son statut: l'actant devient une pure composition modale. Sur la base définitionnelle simple de la modalité comme modification de la relation entre un sujet et son prédicat (par le croire, le vouloir, le devoir, le savoir, le pouvoir, et leurs négations), chaque actant du discours - qu'il s'agisse d'un personnage de récit ou d'un concept philosophique engagé dans un faisceau de prédicats - est littéralement constitué par son équipement modal. C'est ainsi que le Destinateur est défini par la factitivité : tout actant qui fait croire, fait vouloir, fait savoir, fait pouvoir et finalement fait faire reçoit cet habillage particulier du Destinateur dit «manipulateur ». L'élargissement est immédiat : l'activité persuasive est un des attributs essentiels du Destinateur et la rhétorique apparait globalement, autour de la manipulation cognitive et pragmatique, comme relevant de sa sphère. Une analyse comparable, en termes modaux, peut être appliquée aux autres actants, évolutifs et modulables, chaque acteur pouvant alternativement ou simultanément revêtir plusieurs statuts actantiels. On comprend dès lors pourquoi on a pu parler de la « charpente modale du sens » (Brandt, 1992).

13 Le premier des Entretiens de Confucius $(1987$, p. 9) vient illustrer cette extension de l'ordre narratif du discours hors du genre proprement narratif :

Le Maître dit : « N'est-ce pas une joie d'étudier, puis, le moment venu, de mettre en pratique ce que l'on a appris? N'est-ce pas un bonheur d'avoir des amis qui viennent de loin ? Et n'est-il pas un honnête homme celui qui, ignoré du monde, n'en conçoit nul dépit?»

Indépendamment du contenu de chacune des questions, c'est leur succession qui fait énigme et se présente comme un problème à la lecture de cet "entretien ». Ses énoncés sont-ils disjoints, séparés par quelque abyssal silence sémantique? Si ce n'est pas le cas, quel principe sous-jacent en assure la liaison et la cohérence ? Or, en interrogeant au plus près du texte la trame potentielle qui les lie, on peut considérer que leur enchainement repose sur un schème narratif au sens étendu que la sémiotique propose. En effet, la première question indique le contrat et les valeurs qu'il vise, à travers la performance (" étude et mise en œuvre ») ; la deuxième présuppose la performance réalisée, sous la forme élémentaire du déplacement dans l'espace et des épreuves rencontrées qui consolident et intensifient la valeur ( « des amis qui viennent de loin »); et la troisième $a$ trait à la dernière étape du schéma narratif, celle de la reconnaissance, sous la forme ici également valorisée du « renoncement " à la gloire et de la « sérénité », sagesse ultime. Il s'agit là d'une hypothèse analytique sans doute, mais elle montre comment les trois questions s'articulent l'une avec l'autre suivant un ordre qui n'est autre qu'une proposition narrativisée de forme de vie. 


\section{La narrativisation de l'énonciation}

15 L'énonciation, provisoirement rejetée - pour des raisons méthodologiques, et en vertu du principe d'immanence - de l'approche sémiotique de la signification (Greimas, 1996, p. 153-154) ${ }^{4}$, a en réalité fait un retour très important dans la problématique d'ensemble, notamment à travers sa narrativisation. Avant d'en donner un exemple fameux dans l'histoire de la littérature, indiquons en quelques mots les grands paramètres de l'énonciation tels qu'on peut les envisager d'un point de vue sémiotique.

16 Tout d'abord, l'énonciation individuelle, celle de la parole en acte, est rapportée à l'énonciation collective qui la rend possible: elle émerge de la masse parlante qui façonne, au fil des générations et de leur multitude d'actes énonciatifs, la langue telle que nous en héritons et telle, inévitablement modifiée, que nous la transmettrons. Cette "praxis énonciative » engendre des formes qui se figent et se sédimentent, que nous appelons l'usage. Chaque énonciateur individuel convoque ces formes - et c'est toute la part d'automatismes langagiers lentement acquis par l'apprentissage qui se déroulent ainsi de bouche en bouche depuis la phraséologie et les lieux communs jusqu'aux attentes génériques. Le créateur de langue - individuel par idiolecte, collectif par sociolecte révoque quant à lui ces produits de l'usage, et «invente» des formes nouvelles. L'écrivain, comme le disait M. Proust repris par G. Deleuze, «écrit dans une sorte de langue étrangère $»$.

17 L'acte en lui-même est analysé en une double opération, dite de débrayage - la fabrique $\mathrm{du}$ «il/elle, ailleurs, autre temps» - et de l'embrayage - la fabrique du «je, ici, maintenant ». L'embrayage, discours à la première personne des récits autobiographiques comme du registre lyrique, présuppose, dans l'ontogenèse, l'existence du débrayage : on apprend le «il» avant le «je », et sa maitrise est même une condition du discours à la première personne. La boutade d'A. J. Greimas est connue : « "Il" est, après le cheval, la plus noble conquête de l'homme ».

Enfin, troisième étage de l'édifice énonciatif, pas de discours en acte qui ne doive, à l'instar de la perception elle-même, se soumettre aux contraintes de la perspective - c'est elle qui nous fait adopter en lisant un roman le parcours du criminel ou celui du policier. Celle-ci se met en place, du côté du sujet, à travers la vaste problématique du point de vue, et du côté de l'objet, à travers celle, non moins complexe, de la focalisation. Double impératif de la perspective qui ouvre, du fait des contraintes inhérentes à la textualisation, cette itération de l'énonciation liée à la linéarité spatio-temporelle de toute textualité - un vaste espace stratégique pour l'auteur qui détermine notre condition de lecteurs.

Un auteur justement, M. de Cervantès, vient à point nommé pour illustrer, parmi ces ouvertures, le champ narratif de l'énonciation elle-même. Les aventures de Don Quichotte, avec ses cent vingt-six chapitres en deux tomes, constituent bien entendu un formidable vivier de narratologie. Tous les romans de chevalerie y sont mis en abyme pour nourrir le roman de son héros et de son écuyer; et à côté de cet ensemble, il y a le roman de la nièce et de la gouvernante, ceux du curé et du barbier, ceux de l'aubergiste et de toute la population des gens "normaux » dont on s'aperçoit, lorsqu'ils se rencontrent ou se racontent, qu'ils sont peu ou prou pris dans la même folie narrative que le héros luimême. Or, le socle immuable des innombrables récits intercalés, portés par des narrateurs tout aussi nombreux, empruntant en prose ou en vers tous les genres narratifs 
disponibles à l'époque, allant même, pour un chanoine de rencontre, jusqu'à un métadiscours critique et savant sur le roman de chevalerie alternativement blâmé et comblé d'éloges (t. 1, chap. 47 et 48 ), ce socle n'est autre que le schéma narratif canonique. Il suffit de relire la trame du rêve de don Quichotte lui-même dans le chapitre premier pour en apercevoir les séquences immuables : épreuve qualifiante, décisive et glorifiante, ou (auto-)manipulation - action - sanction (positive bien entendu).

Il lui vint la plus étrange pensée que jamais fou ait pu concevoir. Il crut bon et nécessaire, tant pour l'éclat de sa propre renommée que pour le service de sa patrie, de se faire chevalier errant, et d'aller par le monde avec ses armes et son cheval chercher les aventures, comme l'avaient fait avant lui ses modèles, réparant, comme eux, toutes sortes d'injustices, et s'exposant aux hasards et aux dangers, dont il sortirait vainqueur et où il gagnerait une gloire éternelle. (Cervantès, 1997, p. 57)

Or, parallèlement, un autre univers narratif beaucoup plus complexe et tout aussi multiple se trame. C'est celui de l'énonciation narrative elle-même, qui prend en charge la dérision du récit, la dérision de tout dire narratif.

La voix qui porte ce festival de l'illusion est inassignable. Plus précisément, elle multiplie les moyens d'échapper à toute assignation. Don Quichotte est le récit d'un narrateur qui toujours se dérobe, niant sa position énonciative et la faisant transiter d'énonciateur en énonciateur. De ce point de vue, son texte a une structure fractale : il plonge dans la machinerie récursive de la parole en acte. Le Prologue, que l'auteur s'avoue impuissant à écrire, est pris en charge par un ami bienveillant qui lui dicte les techniques du plagiat. Dès la deuxième partie, le manuscrit s'étant perdu au beau milieu d'un combat - la bataille contre le « courageux Biscayen »-, le texte espagnol n'est plus que la traduction de vieux cahiers en arabe, trouvés sur un marché à Tolède, écrits par "Sidi Ahmed Benengeli, historien arabe ». Le « je » de l'énonciateur embrayé surgit çà et là, comme par accident, et le plus souvent la parole narrative est déléguée par tous les moyens à un autre, à n'importe quel autre. Plus encore, la réalité éditoriale vient, avec le second tome, s'entremêler avec la fiction. En 1614, un an avant la publication de la suite des aventures de don Quichotte dont le premier volume date de 1605, parut à Tarragone l'œuvre d'un faussaire sous le même titre, vendue comme le deuxième volume des aventures du célèbre chevalier. En interpellant son lecteur dans le prologue du second tome, véritable, «l'auteur ", si c'est lui, déclare : " Tu voudrais peut-être que je traite cet homme-là d'âne, de sot, d'impertinent? Eh bien sache que je n'en ai pas la moindre intention.» Et il intègre le personnage plagié au récit des nouvelles aventures, don Quichotte rencontrant alors des lecteurs de la fiction dont il est issu, ou renonçant à se rendre dans telle ville parce que son double de papier y avait séjourné...

Cette vertigineuse mise en abyme de l'énonciation, métadiscursive de bout en bout, constitue un plan de composition énonciative qui a pour objet, non le dit, mais le dire avec ses contraintes narratives. Car cette dérobade continue face à l'énonciation du récit, toujours inexorablement pourvoyeur d'illusions, ne peut continuer à s'énoncer qu'en consentant, envers et contre tout, aux contraintes immanentes de la narrativité. Pour définir ce régime d'immanence, nous dirons qu'il consiste à ne pas se faire d'illusion sur l'illusion. Il se trouve pris, par sa récursivité même, dans le cercle vertigineux d'un illusionnisme narratif dont on ne saurait sortir.

C'est ainsi que Don Quichotte de M. de Cervantès se trouve finalement soumis, en raison de la pluralisation énonciative des positions narratives, à l'impératif de la narrativité au sein de l'énonciation elle-même, condition de possibilité de son dire. Le roman s'ordonne donc 
à travers deux régimes narratifs distincts : l'un reposant sur le plan de la technique du récit où se déposent les séquences, purs produits stéréotypés de l'usage et de la culture des histoires, et l'autre se situant sur ce que G. Deleuze et F. Guattari (1991, p. 185) appellent le "plan de composition esthétique ", celui qui travaille le matériau et le fait advenir - ici le matériau énonciatif - pour imposer le composé sensible qui rend «la matière du texte expressive $\mathrm{e}^{5}$ ». C'est bien le phénomène qui se produit avec l'énonciation narrative chez $\mathrm{M}$. de Cervantès. Elle le conduit de la narratologie critique à la narrativité irréductible. On peut constater que la construction technique de la narration remonte dans le matériau qui est l'acte de son énonciation, et que celle-ci devient, pour le lecteur, le foyer de ses sensations. C'est le geste d'écrire et de narrer (traduire, copier, plagier, déléguer, multiplier les simulacres de parole, faire bégayer les formes narratives) qui se trouve confronté aux implacables contraintes narratives du sens.

\section{Le narratif et le passionnel} se définit comme transformation des états de choses ou des états de personnes : pauvreté devient richesse, et servante devient reine. Mais le statut des sujets d'état impliqués dans la transformation ne se limite évidemment pas à la nouvelle forme de "jonction" statique dont il résulte. Ce statut est lui-même mouvant, fluctuant et modulable : le sujet est sensibilisé par la jonction. Même glorifiée, Cendrillon conserve peut-être une certaine amertume de l'humiliation que sa belle-mère et ses demi-sœurs lui avaient infligée. Gilliatt le malin, héros des Travailleurs de la mer de V. Hugo (1866), en sauvant la machine de La Durande, échouée sur les Roches Douvres, a bien gagné la main de Déruchette dont il est épris, promise par Mess Lethierry, tuteur de la jeune fille et armateur du navire. Il y renonce pourtant avec abnégation, sacrifie son bonheur à l'amant que Déruchette lui préfère, et accomplit l'acte suicidaire que son désespoir lui dicte. On le voit, le passionnel nait du récit, il est issu de sa trame ou du moins coextensif avec elle.

Une des originalités de la sémiotique est ainsi d'avoir étroitement associé les problématiques de l'émotion et de la passion avec celle de l'action et de la narrativité. Comme l'écrit P. Fabbri (2008) : « La narrativité est radicalement un acte de configuration $\mathrm{du}$ sens à travers des actions et des passions.» Loin de réduire le narratif à l'enchainement des actions, principe longtemps admis comme la clef de toute modélisation dans ce domaine, la sémiotique assume l'entrelacs qui associe le passionnel au narratif. Cela implique que l'un ne peut être ni pensé ni articulé sans l'autre et que les dispositifs affectifs sont consubstantiels à la structure narrative. Les transformations de l'agir affectent les sujets et les passions des sujets ne prennent forme que dans la dimension programmatique du récit.

C'est donc la jonction elle-même qui, à travers ses modes d'existence variés, est sensibilisée, formant alors la structure d'accueil des passions: l'impatience est une sensibilisation de la conjonction attendue, encore virtuelle; la nostalgie est l'émotion d'une conjonction irrémédiablement révolue; l'obstination est l'état sensible d'un sujet souffrant d'une disjonction qui s'accentue au fur et à mesure qu'il tente de la transformer; l'enthousiasme est à l'intensité extrémale de la conjonction ce que le désespoir est, de la même manière, à la disjonction... Aussi sommaire que cette approche puisse paraitre en raison du caractère formel de la description qu'elle propose, elle indique clairement le lieu où les dimensions sensible, émotionnelle et passionnelle peuvent être prises en compte, indépendamment de toute considération proprement

Pratiques, 181-182 | 2019 
subjective. Ce lieu, dans le discours, est celui de la syntagmatique narrative, source possible de la paradigmatisation des passions et de leur typologie.

C'est ainsi que l'hypothèse d'un "schéma passionnel ", qui ordonnerait en sous-main la mise en forme et en discours des parcours affectifs, est parallèle au schéma narratif bien connu. A. J. Greimas et J. Fontanille (1991) mettent en évidence cette large syntagmatique des passions, en l'inscrivant dans une suite de quatre séquences: 1 . la disposition, qui indique la structure d'accueil, en son état virtuel, qu'offre le sujet à telle ou telle forme passionnelle (cf. en psychologie, le «caractère» ou le "tempérament»); 2. la sensibilisation, qui actualise cette disposition, à l'occasion d'un évènement, d'une perception ou même d'une sensation (qu'on pense au "léger trouble " qu'éprouve la Princesse de Clèves en apercevant le duc de Nemours) ; 3. l'émotion, cœur du parcours et moment de la crise, lorsque l'évènement fait rage et que la passion manifeste somatiquement ses excès par l'agitation, la paralysie, les palpitations (cf. Phèdre: «J'aime... A ce nom fatal, je tremble, je frisonne/ [...] Je le vis, je rougis, je pâlis à sa vue ») (Racine, 1677, acte 1, scène 3); 4. la moralisation enfin, lorsque, la crise passée, le sujet réintègre l'ordre social de la mesure, et que son monde axiologique se réinscrit dans celui des règles collectives (cf. la «muflerie » de l'amant qui forme le desinit d'Un amour de Swann: «Dire que j'ai gâché des années de ma vie, que j'ai voulu mourir, que j'ai eu mon plus grand amour pour une femme qui ne me plaisait pas, qui n'était pas mon genre! » - nous soulignons) (Proust, 1988, p. 375). Cette «moralisation» finale sanctionne le parcours, à l'instar de la phase de « reconnaissance » du schéma narratif, de même que l'émotion en constitue l'épreuve et que les disposition et sensibilisation coïncident avec le contrat et l'instauration de la compétence du sujet.

Mais la forme la plus cruciale de cette convergence entre la narrativité et la signification passionnelle se situe autour des « simulacres » générés par la perception et l'énonciation passionnées. Les simulacres sont les formes-écran que le sujet installe entre lui et son objet, ou plutôt que lui renvoie cet objet, et sur lesquelles il fonde ses espérances ou ses craintes, ses désirs ou ses dégoûts. «Considère mon amour avec quel excès tu as manqué de prévoyance. Tu as été trahi et tu m'as trahie par des espérances trompeuses... » s'écrie, dans l'incipit de sa première Lettre, la Religieuse portugaise en s'adressant à son propre sentiment (Anonyme, 1993). Car le sujet passionné actantialise ces formes nées de son affect et transforme ses simulacres en de véritables personnages, dotés de compétences et de capacités d'action. La moralisation des simulacres les interprète comme manifestations de l'«aveuglement» propre à la passion. Mais ils constituent aussi un ressort narratif essentiel.

Lorsque le narrateur de La Recherche s'apprête à révéler à son ami Robert de Saint-Loup l'image d'Albertine, être céleste de sa passion, sous la forme référentielle d'une photographie, il tremble de l'écart entre les deux perceptions possibles. Et il n'a pas tort :

" Elle est sûrement merveilleuse ", continuait à dire Robert, qui n'avait pas vu que je lui tendais la photographie. Soudain il l'aperçut, il la tint un instant dans ses mains. Sa figure exprimait une stupéfaction qui allait jusqu'à la stupidité. «C'est ça la jeune fille que tu aimes?", finit-il par me dire d'un ton où l'étonnement était maté par la crainte de me fâcher. Il ne fit aucune observation, il avait pris l'air raisonnable, prudent, forcément un peu dédaigneux qu'on a devant un malade eût-il été jusque-là un homme remarquable et votre ami - mais qui n'est plus rien de tout cela car, frappé de folie furieuse, il vous parle d'un être céleste qui lui est apparu et continue à le voir à l'endroit où vous, homme sain, vous n'apercevez qu'un édredon. (Proust, 1925, p. 30) 
30 l'enveloppant d'une «stratification de sensations » inaccessibles à son ami, synesthésie sensorielle et affective faite d'odeurs, de saveurs et d'autres sensations plus « indéfinissables » s'ajoutant à la vision, et qui font qu'Albertine est pour lui, «comme une pierre autour de laquelle il a neigé, que le centre générateur d'une immense construction qui passait par le plan de mon cœur » (ibid., p. 31).

31

simulacres passionnels sont les produits narratifs de ces «sensations interposées entre le visage de la femme et les yeux de l'amant - l'énorme œuf douloureux qui l'engaine et le dissimule autant qu'une couche de neige une fontaine " (ibid.). De leur formation et des configurations qu'ils génèrent dépendent non seulement le cours narratif du récit avec ses stratégies et ses antagonismes, mais aussi, comme le montre la citation qui précède, les agencements figuratifs du discours, mêlant les métaphores aux représentations effectives, multipliant les déformations, ouvrant de nouvelles perspectives à la lecture et à l'interprétation, associant les registres, comme par exemple la distanciation humoristique au pathétique passionnel. Il en va ainsi de cette rencontre de « l'être céleste » et de « l'édredon » - générateurs à leur tour d'histoires disjointes.

\section{Perspectives}

32 Le paradigme greimassien de la narrativité, dont on vient de résumer quelques aspects essentiels à nos yeux, semblait donc s'être affaibli avec le développement des problématiques qui lui ont succédé - celle de l'énonciation, celle des passions et celle de la perception -, alors même que celles-ci se nourrissaient de celui-là. Il se trouve simplement que le récit avec les différents genres et formes de la narratologie n'a plus été, en tant que corpus de discours, au centre des préoccupations sémiotiques.

Car la dynamique de la recherche suscitée par le paradigme greimassien a suivi de nouvelles orientations. On peut distinguer, dans le seul champ français, et sans préjuger d'autres développements théoriques en Italie, en Belgique ou dans l'univers latinoaméricain, cinq grandes voies de transformation de la sémiotique: sémiotique tensive (Zilberberg), sémiotique des pratiques (J.Fontanille), sémiotique des instances énonçantes (J.-C. Coquet), sémiotique des interactions (É. Landowski) et sémiotique de l'iconicité (J.-F. Bordron). À chacune se rattache le nom d'un chercheur qui s'y est plus particulièrement investi. En dépit de leurs différences et parfois de leurs antagonismes, elles sont toutes les cinq, à des degrés divers, héritières du «tournant phénoménologique » de la discipline à la fin des années 1980, c'est-à-dire de l'irruption du sensible (perceptif et émotionnel) dans la saisie du sens. Il n'est évidemment pas question de les présenter ici de manière approfondie. Nous souhaitons seulement montrer, en les localisant, comment les propositions de deux d'entre elles (sémiotique tensive, sémiotique des instances) s'intègrent à la trame narrative du sens et l'enrichissent. Loin donc de tout souci d'exhaustivité, on n'en retiendra que quelques aspects significatifs, dans la mesure où ces voies présentent aussi des ouvertures et des contributions à l'analyse du récit. 


\section{Sémiotique tensive et logique de l'évènement}

34

L'ouvrage de référence dans ce domaine est l'essai de C. Zilberberg, Éléments de grammaire tensive (2006). L'« hypothèse tensive », comme aimait à la nommer son promoteur, s'inscrit clairement dans la tradition structurale et ses positions fondatrices. Elle reconnait le primat de la différence et de la relation, mais elle l'appréhende en termes de dépendance, c'est-à-dire de rection et donc de hiérarchie. Cette hypothèse consiste à dépasser la saisie de la discontinuité impliquée par l'identification des catégories - le fameux Vs-versus -, et postule le caractère continu - mouvant, tensif et intensif - de la signification. Elle considère que la transformation commence par les variations d'intensité et que la rupture, le changement de valence - le radical passage d'un état à un autre par exemple - n'en est qu'un des cas de figure.

La question initiale de C. Zilberberg dans ce sens, qui a donné son titre à un de ses plus anciens articles, fondateur de l'approche tensive, était: "Sous les sèmes, y'a quoi ?» (Zilberberg, 1981). Le sème étant l'élément minimal discrétisé de la structure sémantique, il s'agissait d'en rechercher le continuum sous-jacent. Ce faisant, cherchant la modulation sous les marques déposées dans la langue, la sémiotique tensive postule le primat de l'affect et de l'expérience sensible. Celle-ci, du reste, résultant de la praxis énonciative est déjà inscrite dans le lexique. Ainsi, en français, sous l'opposition chaud/froid se trouvent les modulations graduelles de la variation thermique (tiède, etc.) qui culminent dans le «brûlant » et le "glacé », lesquels impliquent non seulement l'identification objective d'une qualité, mais la relation sensible avec le moi-peau !

6 Cette élasticité sémantique au sein de la catégorie conduit C. Zilberberg à identifier des sous-contraires et des sur-contraires, des termes « atones » et des termes «toniques ». Or, et c'est là une des originalités à nos yeux les plus intéressantes de cette approche, la relation, selon le degré d'intensité, implique des formes de rationalité et, on va le voir, de narrativité, différentes. Ainsi, nous avons l'opposition classique "ouvert» versus "fermé », qu'il identifie comme sous-contraires; chacun des termes s'intensifie en un sur-contraire : d'un côté le " béant », de l'autre l'« hermétique ».

Or les deux ordres de relation renvoient à deux logiques distinctes: les sous-contraires sont unis par la logique de l'implication, du classique syllogisme au «si... alors ». « Si la fenêtre est ouverte, alors je peux la fermer » et inversement. L'hermétique et le béant ne répondent pas à cette logique: F. Ponge nous dit que "l'huître est un monde opiniâtrement clos "... si bien hermétique qu'on ne peut pas l'ouvrir. De même, si une plaie est béante, on ne peut la refermer. C'est là qu'intervient l'autre logique, propre aux sur-contraires et plus largement aux positions toniques : cette logique est celle de la concession, que les grammairiens commentent comme cause inopérante (bien que..., quoique..., alors que..., même si..., malgré tout..., quand même..., etc.). «Bien que cette la plaie soit béante, je la ferme », proclame le chirurgien intrépide, «bien que cette cause soit indéfendable, je la prends en charge ", dit l'avocat péremptoire. Ainsi, le concessif s'oppose à l'implicatif comme le survenir au devenir. L'intérêt de considérer à la même hauteur conceptuelle ces deux formes de raisonnement consiste à restaurer la place du sensible dans le cognitif: voici qu'apparait sous un autre jour le problème de la nouveauté, issue d'un geste concessif (bien que ce soit inattendu, cela arrive!), et les passions de la surprise (l'admiration, subite surprise de l'âme, est selon R. Descartes, rappelons-le, la première de toutes les passions). Plus encore, c'est le statut de l'évènement 
lui-même qui s'éclaire : dans la masse quotidienne des faits, combien peuvent être isolés, sélectionnés et reconnus comme des évènements, survenant dans la morne prévisibilité du quotidien ? Son fondement est concessif : alors que rien ne le laissait prévoir... Et voici qu'il change le sens de ce qui le précédait et le sens de ce qui le suit, voici qu'il impose une accélération du tempo, voici qu'il intensifie la perception, voici qu'il génère des valeurs et sensibilise le sens au point de le transformer en passion (peur, enthousiasme, désespoir, etc.).

Or, force est de reconnaitre que le concessif recouvre lui aussi deux formes, l'une tonique - celle que l'on vient de suggérer - et l'autre, atone, qui prend forme dans l'argumentation, dans la négociation, et résulte du tâtonnant partage des valeurs, dans le donnant-donnant : « certes... mais... » : je vous concède, je fais des concessions.

Cette logique du concessif (tonique) ouvre de larges perspectives, notamment dans l'ordre narratif. En effet, on comprend que le schéma narratif greimassien soit une version généralisable certes, universelle peut-être, d'un imaginaire du « sens de la vie ». Mais il est clairement adossé à la logique implicative du devenir : " tu seras un homme mon fils, si... si..., si... alors..., alors..., alors... ». De contrats en compétences à acquérir, de compétences formées en épreuves traversées, puis d'épreuves en sanctions négatives et positives, le devenir ou même le parvenir du héros se forme peu à peu au fil d'une existence programmée. Le schéma concessif tonique du survenir engendre quant à lui un tout autre schème narratif, massivement exploité dans l'univers des récits (textuels et cinématographiques), dans le champ politique comme dans celui des récits médiatiques. Le genre de la nouvelle y puise une de ses règles principales, la narration de la catastrophe y trouve ses principes moteurs et le cinéma hitchcockien s'en est fait une marque qui est plus que stylistique: c'est la violente attente de l'inattendu, c'est l'hypertrophie de l'évènement comme pivot narratif, c'est le schème du survenir et du suspense, qui ne présuppose que dans l'après-coup, impose des catalyses narratives improbables à partir de qui est survenu (c'est-à-dire des reconstructions logicosémantiques de séquences antérieures) et génère, de gré ou de force, la nouveauté.

\section{Sémiotique des instances et infra-narrativité}

L'auteur de référence dans ce domaine est J.-C. Coquet, et notamment son livre Phusis et logos (2007). Dans le prolongement de cette même conception, citons aussi un numéro spécial de la revue Littérature sur le thème: "Comment dire le sensible? Approches sémiotiques » (Bertrand \& Coquet, 2011).

41 Il est difficile, ici comme précédemment, de résumer cette approche en quelques mots, mais on peut dire que son fil rouge consiste à rapporter la signification à l'instance qui l'énonce. Elle pose donc l'énonciation comme son concept directeur et a pour référence principale les travaux d'É. Benveniste. C'est dire qu'elle se met à distance du principe d'immanence, le considérant comme une construction abstraite coupée de l'expérience vive du sens en acte, et elle lui oppose le principe de réalité. Par là, cette approche assume une double condition signifiante, à la fois langagière et phénoménologique: "Par l'énonciation, mais aussi par la perception nous nous conjoignons au monde ». Lorsqu'il s'est positionné de manière polémique par rapport à A. J. Greimas, J.-C. Coquet aimait qualifier son approche de « subjectale » par opposition à une sémiotique « objectale». Les développements de cette approche qui met le sujet au centre ont été particulièrement 
importants, ce qui n'est pas pour surprendre, en psycho-sémiotique (autour des travaux du chercheur I. Darrault).

Au regard de ce qui nous intéresse ici, c'est surtout le dispositif des instances énonçantes qui conduit à un nouveau regard sur le récit. En effet, J.-C. Coquet élabore un dispositif actantiel élémentaire (entre sujet, objet et tiers-actant - ou Destinateur) au sein duquel il distingue le sujet du non-sujet. Le sujet est celui qui prédique et qui assume son discours, il est celui qui «se dit je »; le non-sujet est celui qui prédique sans assomption, celui qui « récite sa leçon » soumis à l'impersonnel de l'énonciation, ou qui énonce en automate et ne réalise que les actes pour lesquels il a été programmé (comme le loup de la fable qui cherche en vain à s'arracher à son état de non-sujet en prétendant faire valoir les raisons de son acte, inéluctablement erronées); c'est encore, selon J.-C.Coquet, le sujet passionnel, soumis à la loi impérieuse de ses objets, qui ne peut s'arracher à la passion que par l'assomption qui lui permet de la maitriser et de se réhabiliter comme sujet (le sémioticien renouvelant ainsi la catégorie passion/raison, dont l'approche précédente, greimassienne, s'était détachée en valorisant la catégorie définitoire passion/action). Par ce biais du non-sujet de la passion, l'opposition entre la phusis et le logos fait son entrée. Relèvent de la phusis les prédicats somatiques du sujet sensible, celui de la perception et de l'émotion, par lesquels ils affirment leur prise sur le monde. Le logos, d'ordre cognitif, est second par rapport à la phusis et exprime la reprise, à la fois intellectuellement distanciée, déformée et contrôlée par le savoir. «Les prédicats somatiques (ou de réalité) expriment le sensible, écrit J.-C. Coquet, alors que les prédicats cognitifs décrivent le monde."

À cela s'ajoutent selon nous, dans le dispositif des instances énonçantes, les thématisations des actants (qui fondent les "rôles thématiques »). Celles-ci, définies comme codification des instances et plus précisément mode d'insertion de l'individuel dans le collectif - " père ", «mère ", " étudiant ", « président ", « loup ", « citoyenne ", etc. -, déterminent en chaque énoncé le statut du dire, depuis ses praxèmes et ses registres jusqu'à ses modulations signifiantes (tempo, prosodie, etc.).

À travers les diverses complexifications de l'énonciation en scènes d'instances, l'univers narratif se trouve à son tour enrichi et affiné. La scénographie des instances a fait irruption dans l'espace du récit avec les Tropismes de N. Sarraute et les micro-drames de l'infra-conversation. Distinguées selon leurs modes d'existence, virtuel ou actualisé, les instances sont en compétition pour la manifestation. Telle ou telle, normalement masquée dans un jeu de rôle thématique socialement réglé, peut surgir inopinément sous la forme d'un lapsus, révélant le bouillonnement instanciel sous l'apparence d'une énonciation contrôlée (cf. l'espace thématique réglé des rôles politiques par exemple, où surgissent les lapsus). Le réglage de ces rôles est le produit d'un long apprentissage et d'une patiente codification de la praxis énonciative.

On prendra pour conclure et pour illustrer la portée narrative de ces jeux d'instances, deux exemples de leur architecture dans le champ littéraire. Le roman, au XIX ${ }^{\mathrm{e}}$ siècle, est, comme on le sait, épris de science et de technique. Pour ne prendre que le cas des romans terraqués, qu'il s'agisse d'E. Poe dans « Descente dans le Maelström », de V. Hugo dans Les Travailleurs de la mer, d'H. Melville dans Moby-Dick ou de J. Verne dans Vingt mille lieues sous les mers, tous exploitent les différents champs du savoir de leur époque et en assument les données discursives premières: le faire paradigmatique des classifications, des taxinomies et des typologies. Comparons à ce sujet J. Verne et H. Melville. 

(deux décennies après la première édition), ce texte scientifique d'une soixantaine de
pages ne semble pas avoir un statut narratif clair, et son lien avec le roman
n'apparaissant pas au premier éditeur, il avait été tout bonnement exclu. Se présentant comme un véritable archipel des savoirs, à la manière d'un programme d'une École des hautes études, il met en œuvre et en scène toutes les disciplines des sciences humaines et sociales (la philologie, l'histoire des religions, la science politique, l'Histoire, etc.) et des sciences exactes (la botanique, la météorologie, la géologie, la biologie, la technologie, etc.), chacune disposant d'un chapitre spécifique. Mais le discours n'est pas seulement scientifique, et c'est l'énonciation elle-même qui se révèle narrativisée. Ainsi, une autre instance énonciative double l'instance cognitive, c'est celle de l'écrivain ou du poète. Le lecteur constate en effet que l'écriture forme en elle-même, transversale aux domaines des contenus dont elle est le véhicule, un objet propre. Après un chapitre inaugural à caractère mythique, voici qu'on assiste à la naissance d'une langue : le chapitre suivant est uniquement constitué en effet de courtes phrases nominales; le suivant de propositions indépendantes avec des verbes élémentaires (copules et modaux) ; le suivant avec des phrases plus complexes et des verbes d'action; le suivant avec des paroles citées; le suivant avec des personnages du pays et des paroles dialoguées... Ainsi, de chapitre en chapitre, la langue s'étoffe et accompagne la naissance de ce monde insulaire que l'exilé découvre et dont il fait le centre de son univers. Le matériau-langage se fait matériau-hommage pour exalter, ce sera la dédicace de jonction avec le roman lui-même, l'accueil que les habitants de cette petite ile anglo-normande ont fait au proscrit de « Napoléon le petit ». Ainsi, le récit entre dans l'écriture, et c'est l'énonciation elle-même, avec son jeu d'instances énonçantes, qui en forme la scène. Cette propriété qui consiste à faire retour sur le langage et en exhiber le matériau, relevant typiquement de l'action 
littéraire, nous met alors bien loin du discours référentiel que Jules Verne tiendra en l'entrecroisant méthodiquement, mais sans le mélanger, avec le discours fictionnel.

De la même façon, H. Melville fait dialoguer, dans Moby-Dick, la littérature avec la science. Cela se passe, notamment, dans le chapitre XXXII de son roman, intitulé «Cétologie » que nous avons évoqué plus haut. Il y présente, en bon naturaliste disciple du savant Lamarck, une taxinomie détaillée des cétacés, sur le fond de toile de la théorie transformiste, en attendant l'œuvre de C. Darwin (Sur l'origine des espèces, où apparait la théorie de l'évolution, est publié en 1859). Or, de manière apparemment surprenante, les critères qui fondent cette taxinomie se réfèrent à l'univers du livre et de l'édition - et donc, en arrière-plan, au langage lui-même. Les cétacés sont en effet classés par dimension décroissante - du grand cachalot au modeste marsouin - selon le pliage des feuilles de papier pour la fabrication des livres : in-folio, in-octavo, in-douze, chacune de ces grandes classes définissant des « livres » qui se subdivisent en « chapitres » où entrent les types et les espèces. Savoirs croisés, rapportés à l'écriture et à la communication. La nature entre dans l'ordre du langage à travers sa modeste grammaire matérielle, celle de la fabrication des livres. Le pliage peut alors être compris comme métaphore du lexique et des récits qui déploient les noms.

Ou plutôt : la nature est pliée dans notre langage. Et ce pli est si prégnant et si décisif dans l'écriture de H. Melville qu'il s'ouvre, au terme de la taxinomie, sur une interrogation concernant le pliage du monde par les mots et sur leur essentielle relativité. La confrérie des Léviathans en effet se prolonge au-delà des classes et des types connus et identifiés jusqu'ici dans le régime taxinomique adopté. Le narrateur en effet raconte :

En se référant à des sources islandaises et hollandaises, ou à de vieux auteurs anglais, on pourrait dresser d'autres listes de baleines incertaines, qui ont le bonheur de porter les noms les plus invraisemblables. Je les crois totalement obsolètes et m'abstiendrai donc d'en faire état ; j'irai même jusqu'à les soupçonner de n'être que des sons, pleins de fureur léviathanesque, mais qui ne signifient rien. (Melville, 2006, p. 170)

51 De purs signifiants, donc. Qui ouvrent sur l'océan du monde innommé et innommable à la surface duquel flottent nos précaires petits drapeaux lexicaux. Et le chapitre scientifique qui s'est imperceptiblement transformé en un grand récit épistémologique s'achève sur l'inachèvement, comme condition même de l'œuvre. Nous retrouvons ici la sémiotique : les lecteurs de De l'imperfection d'A. J. Greimas feront aisément le lien entre les deux textes qui se rejoignent dans la réflexion aspectuelle sur l'imperfectif comme condition de l'esthétique, deux textes que presque un siècle et demi sépare.

Je laisse mon système cétologique inachevé, comme est demeurée inachevée la grande cathédrale de Cologne, avec sa grue oisive au sommet de la tour incomplète. Car si les petits édifices peuvent être terminés par leur architecte primitif, les grands, les vrais, confient toujours la pose du couronnement à la postérité. Dieu me garde de jamais rien parfaire! Ce livre tout entier n'est qu'une esquisse... même pas : l'esquisse d'une esquisse. Ô Temps, Force, Argent, Patience! (Melville, 2006, p. 170)

Comme chez V. Hugo, le poétique est la raison ultime du sens : cet élan rimbaldien sur lequel se clôt le chapitre le montre. Ce geste d'ouverture indéfinie du sens de l'œuvre est sans doute plus modeste, porté par un peu d'humour et d'autodérision mais, ainsi proclamé, il a son modèle dans l'ouverture indéfinie du langage. L'une parle de l'autre, inexorablement: "on n'achève pas le sens. » La littérature creuse un abîme dans la langue, ou ouvre la langue sur l'abîme, et c'est son récit premier. Comme cela a souvent 
été dit, de M. Proust à G. Deleuze, l'écrivain écrit dans une sorte de langue étrangère, en nomade du langage. Peut-être est-ce l'aventure ultime, un peu vertigineuse, que le lecteur cherche à partager avec l'écrivain, dans la ronde des instances où il se démultiplie.

Quoi qu'il en soit, le développement contemporain de la sémiotique qui fut «narrative et discursive ", désormais implantée comme ici dans les évènements d'énonciation, montre qu'elle est, en définitive, loin de s'écarter du récit. Les conceptualisations du sensible, du passionnel et de la signification en acte, en renouvellent l'approche tout en enrichissant le questionnement narratif sur des fondements théoriques à la fois maintenus, renouvelés et approfondis.

\section{Pour conclure : sémiotique, narrativité, récit}

Il aurait été bien entendu possible de rechercher, comme on l'a fait pour la sémiotique tensive et pour celle des instances, les relations que les recherches sur la sémiotique des pratiques, sur celle des interactions et sur celle de l'iconicité, impliquent pour la production, la lecture et l'analyse des récits. Une synthèse reste à faire, que le volume de cet article déjà long ne permet pas.

Nous pouvons cependant proposer quelques conclusions provisoires.

1. La sémiotique qui s'est définie naguère comme « narrative et discursive » est sortie du champ propre du récit en découvrant que les lois de la narrativité pouvaient être reconnues comme opératoires bien au-delà des seuls genres et formes d'écriture proprement narratifs. Cette généralisation a été la force de son geste théorique, mais aussi sa faiblesse : car si tout discours est narratif, alors le mot «narratif» ne veut plus rien dire.

57 2. La sémiotique, en se détachant des recherches narratologiques, s'est ouverte à d'autres domaines d'investigation - en relation notamment avec les arts plastiques, l'anthropologie et la phénoménologie - tout en restant aussi proche que possible des objets concrets qui forment son domaine d'analyse : les discours. Ces ouvertures et ces investigations offrent néanmoins de nouveaux instruments pour observer, analyser et mieux comprendre le domaine spécifique des récits, littéraires ou non, verbaux ou visuels ou gestuels ou autres.

58 3. Comme on a essayé de le montrer, la réflexion théorique se traduisant en capacité analytique ne conduit pas forcément à une conception totalisante - ce qui a parfois été reproché, non sans raison, à la sémiotique. Les «branches » qui se forment aujourd'hui montrent surtout que les résultats de la recherche sont, par vocation, comme la cathédrale de Cologne, et comme le système cétologique d'Ismael, unique narrateur de Moby-Dick, inachevés et incomplets.

\section{BIBLIOGRAPHIE}

ANONYME (1993) [1669]. Lettres de la Religieuse portugaise. Paris : Librairie générale française. 
BERTRAND, D. \& COQUET, J.-C. (dirs) (2011). « Comment dire le sensible ? Recherches sémiotiques ». Littérature 163.

BRANDT P. A. (1992) [1988]. La Charpente modale du sens. Pour une sémio-linguistique morphogénétique et dynamique. Aarhus : Aarhus University Press.

CERVANTÈs, M. de (1997) [T.1, 1605 ; T. 2, 1615]. L’Ingénieux Hidalgo Don Quichotte de la Manche. Trad. de l'espagnol par A. Schulman, Paris : Éditions du Seuil.

CONFUCIUS (1987) [s. d.]. Les Entretiens. Trad. du chinois par P. Ryckmans. Paris : Gallimard.

COQUET, J.-C. (2007). Phusis et logos. Une phénoménologie du langage. Saint-Denis : Presses universitaires de Vincennes.

COURTÉs, J. (1976). Introduction à la sémiotique narrative et discursive. Méthodologie et application. Paris : Hachette.

Deleuze, G. \& GUATTARI, F. (1991). Qu'est-ce que la philosophie ? Paris : Éditions de Minuit.

FABBRI, P. (2008). Le Tournant sémiotique. Paris : Lavoisier.

GREIMAS, A. J. (1966). Sémantique structurale. Paris : Larousse.

GREIMAS, A. J. (1970). Du sens. Essais sémiotiques. Paris : Éditions du Seuil.

GREIMAS, A. J. \& COURTÉS, J. (1979). Sémiotique. Dictionnaire raisonné de la théorie du langage. Paris : Hachette.

greimas, A. J. \& FontAnille, J. (1991). Sémiotique des passions. Des états de choses aux états d'âme. Paris : Éditions du Seuil.

MELVILLE, H. (2006) [1851]. Moby-Dick ou le Cachalot. Trad. de l'anglais (États-Unis) par P. Jaworski. Paris : Gallimard.

POLKINGHORNE, D. E. (1988). Narrative Knowing and the Human Sciences. Albany : State University of New York Press.

PRousT, M. (1925). À la recherche du temps perdu, t. XIII. Albertine disparue. Paris : Gallimard.

PRoust, M. (1988) [1913]. À la recherche du temps perdu, t. I. Du côté de chez Swann. Paris : Gallimard. RACINE, J. (1994) [1677]. Phèdre, Acte 1, Scène 3. Paris : Larousse.

RICÆUR P. (1983). Temps et récit, t. 1, L'intrigue et le récit historique. Paris : Éditions du Seuil.

RICœUR P. (1984), Temps et récit, t. 2, La configuration dans le récit de fiction. Paris : Éditions du Seuil.

RIC@UR P. (1985), Temps et récit, t. 3, Le temps raconté. Paris : Éditions du Seuil.

SALMON, C. (2007). Storytelling. La Machine à fabriquer des histoires et à formater les esprits.

Paris : Éditions La Découverte.

ZILBERBERG, C. (1981). Essai sur les modalités tensives, Amsterdam ; John Benjamins.

ZILBERBERG, C. (2006). Éléments de grammaire tensive. Limoges : Presses universitaires de Limoges. 


\section{NOTES}

1. La première présentation didactique de la sémiotique, due à J. Courtés en 1976, avait pour titre Introduction à la sémiotique narrative et discursive.

2. Présentation de l'éditeur, publiée sur le site de la revue en ligne Fabula. La recherche en littérature, 16/10/2007.

3. "The core of the argument I make in this book is that narrative is a scheme by means of which human beings give meaning to their experience of temporality and personal actions. [...] Narrative meanings provide a framework for understanding the past events of one's life and for planning future actions. » (Notre traduction.)

4. Voici, pour l'essentiel, le texte de ce rejet : «Tout discours présuppose, on le sait, une situation non linguistique de communication. Cette situation est recouverte par un certain nombre de catégories morphologiques, qui l'explicitent linguistiquement, mais en introduisant dans la manifestation un paramètre de subjectivité, non pertinent pour la description et qu'il faut par conséquent éliminer du texte (à moins que l'analyse n'ait choisi ce paramètre comme objet de description). Ces catégories à éliminer sont principalement: 1. La catégorie de la personne [...]. 2. La catégorie du temps [...]. 3. La catégorie de la deixis [...]. 4. Tous les éléments phatiques en général [...]. »

5. Les auteurs précisent (ibid.): «Le plan technique est nécessairement recouvert ou absorbé par le plan de composition esthétique. C'est à cette condition que la matière devient expressive : le composé de sensations se réalise dans le matériau, ou le matériau passe dans le composé, [...] toujours de manière à se situer sur un plan de composition proprement esthétique. »

6. Le roman de V. Hugo, Les Travailleurs de la mer, est paru en 1865, et celui d'H. Melville, Moby-Dick ou le cachalot, en 1851.

\section{RÉSUMÉS}

Surprise dans le monde des greimassiens en 2014 : voici la narratologie de retour, et en force ! De grands congrès internationaux, réunissant des universités américaines et européennes, sont organisés et se consacrent à l'étude des «fabuleux pouvoirs du récit ». L'ère du storytelling se répand, assurant le succès de l'ouvrage de $\mathrm{C}$. Salmon bien au-delà des frontières de l'université. Or, parmi les références théoriques de ce mouvement de recherche, pour l'essentiel issues du cognitivisme anglo-saxon, pas la moindre allusion à la "sémiotique narrative et discursive ». C'est pourtant sous ce titre que J. Courtés, alors assistant d'A. J. Greimas à l'École de hautes études en sciences sociales, publiait en 1976 chez Hachette un livre d'initiation à la sémiotique. Et dix ans auparavant, dès 1966, le schéma actantiel avait fait son apparition dans Sémantique structurale l'ouvrage fondateur d'A. J. Greimas : la formalisation de la grammaire narrative et son développement en syntaxe modale allaient devenir un des titres de gloire de cette sémiotique. C'est alors que la narrativité, outrepassant les frontières de la narratologie, devait faire son entrée 
dans les sciences humaines et sociales. Et voici qu'elle semblait oubliée. Que s'était-il donc passé ? Ou plutôt, où était passée la théorie greimassienne? Notre propos est ici de faire le point sur cette histoire conceptuelle et de la mettre en perspective. Et nous le ferons en évoquant, en fin de parcours, la filiation descendante de cette théorie, à travers quelques voies sémiotiques qu'elle a enfantées. Non pour en présenter les labyrinthiques conceptualisations mais pour suggérer leurs apports respectifs à une réflexion renouvelée sur le récit.

Surprise in the world of Greimassian scholars in 2014: here is the narratology back, and in force! Major international congresses, bringing together American and European universities, are organized and devoted to the study of the "fabulous powers of narrative". The era of storytelling is spreading, ensuring the success of C. Salmon's book well beyond the borders of the university. But, among the theoretical references of this research movement, essentially derived from Anglo-Saxon cognitivism, not the slightest allusion to "narrative and discursive semiotics". It is nevertheless under this title that J. Courtés, then A.J. Greimas' assistant at the School of Advanced Studies in Social Sciences (École de hautes études en sciences sociales, EHESS-Paris), published in 1976 a book of initiation to semiotics. And ten years earlier, in 1966, the actantial schema had appeared in Structural Semantics, the founding work of A.J.Greimas: the formalization of narrative grammar and its development in modal syntax would become the title of glory of this semiotics. It was then that narrativity, transcending the boundaries of narratology, has been integrated into the humanities. And here it seemed forgotten. What had happened? Or rather, where was the Greimassian theory? Our purpose here is to take stock of this conceptual story and put it into perspective. And we will do so by evoking, at the end of the course, the descending filiation of this theory, through some semiotic paths that it has produced. Not to present the labyrinthine conceptualizations but to suggest their respective contributions to a renewed reflection on the narrative.

\section{INDEX}

Keywords : narrativity, narratology, semiotics, Greimas (Algirdas Julien), modality, enunciation, passion

Mots-clés : narrativité, narratologie, sémiotique, Greimas (Algirdas Julien), modalité, énonciation, passion

\section{AUTEUR}

\section{DENIS BERTRAND}

Université Paris 8 Vincennes-Saint-Denis, LHE, F-93526 Saint-Denis, France 